\title{
Finanzierung nach DRG: Einfluss auf die kardiovaskuläre und pulmonale Rehabilitation
}

\author{
Werden Patienten seit Einführung des DRG-Systems zu früh von Akutspitälern in \\ Rehabilitationskliniken verlegt - mit der Folge einer erschwerten Rehabilitation? \\ Eine Studie in der Klinik Barmelweid versuchte, diese Frage zu klären. Eingeschlos- \\ sen waren 127 Patienten vor und 152 Patienten nach DRG-Einführung.
}

\section{Gilbert Büschinga,}

Frans Hollander ${ }^{b}$,

Christoph Schmidt,

Martin Frey, Johann Steurere

a PT HF, Stv. Leiter Physiotherapie Klinik Barmelweid

b PT-MAS, Leiter Physiotherapie

c Dr. med., Chefarzt Kardiologie

d Dr. med., Leiter Medizinisches Departement Klinik Barmelweid, Chefarzt Pneumologie, alle Klinik Barmelweid

e Prof. Dr. med., Leiter Horten Zentrum, Universität Zürich

Die Arbeit wurde unterstützt vom Forschungsfonds der Klinik Barmelweid, vom Verband physioswiss, der Interessengemeinschaft Physiotherapie Rehabilitation und deren Untergruppe kardiale und pulmonale Rehabilitation.
Korrespondenz: Gilbert Büsching Klinik Barmelweid CH-5017 Barmelweid Tel. 0628572288

gilbert.buesching[at] barmelweid.ch

\section{Einleitung}

Eine Konsequenz des neuen Finanzierungsmodells mit Vergütung nach diagnosebezogener Fallpauschale (Diagnosis-Related Groups, DRG) könnte eine frühere Überweisung von Patienten aus Akutspitälern in die Rehabilitation sein. Als Folge der früheren Entlassung aus dem Akutspital ist theoretisch damit zu rechnen, dass in der Rehabilitation der Anteil an Patienten mit eingeschränkter Mobilität und Eigenständigkeit ansteigen wird. Bei gleichbleibender Rehabilitationszeit könnte damit der Effekt der Rehabilitation abnehmen. Eine Studie aus Deutschland [1] zeigte, dass Patienten früher aus den Akutspitälern in die Rehabilitationseinrichtungen verlegt wurden und der Aufwand beim Pflegepersonal in der Rehabilitation anstieg, aber nicht jeder der beobachteten Effekte konnte in einen kausalen Zusammenhang mit der Einführung der DRG gebracht werden.

Im Rahmen der Begleitforschung haben wir in der Klinik Barmelweid im Jahr vor und nach Einführung der DRG detaillierte Patienteninformationen über den Eintritts- und Austrittsstatus der Patienten erfasst und auf Unterschiede untersucht. Die Fragestellung dieser Beobachtungsstudie ist, ob Patienten früher zugewiesen wurden und sich die Aufenthaltsdauer in der Rehabilitation verändert hat. Dabei untersuchten wir die Leistungsfähigkeit, die Lebensqualität, die Selbständigkeit der Patienten, Komplikationen (inkl. Rückverlegungen) und die Art der Versorgung im Anschluss an die Rehabilitation (ambulante Physiotherapie und Spitex) vor und nach Einführung der DRG.

\section{Methode}

In die Gruppe mit kardiovaskulärer Rehabilitation wurden Patienten mit Status nach einer koronaren Bypassoperation und in die Gruppe mit pulmonaler Rehabilitation Patienten mit einer chronisch obstruktiven Lungenerkrankung (COPD Grad 1-4) eingeschlossen. Erfasst wurden aufgrund der Eintrittslisten alle Patienten, die zwischen Juni und Dezember 2011 (vor Einführung der DRG) und Juni und Dezember 2012 (nach Einführung der DRG) zur kar-

\section{Les effets du financement basé sur les DRG sur la réadaptation cardiovasculaire et pulmonaire}

Avant l'introduction du système DRG au $1^{\mathrm{er}}$ janvier 2012, certains craignaient que cette introduction n'entraîne un transfert prématuré des patients des hôpitaux de soins aigus vers les cliniques de réadaptation, au risque qu'ils ne soient pas prêts pour la réadaptation et que, pour la même durée de séjour en clinique de réadaptation, l'effet de cette dernière soit réduit. La clinique Barmelweid a récolté des informations concernant l'ensemble des patients admis pour une réadaptation cardiovasculaire (suite à un pontage) ou pulmonaire (broncho-pneumopathie chronique obstructive) entre juin et décembre 2011, soit avant l'introduction des DRG, et entre juin et décembre 2012, soit après leur introduction. Cette enquête porte au total sur 127 patients en 2011 et 152 en 2012. En 2012, les patients ont séjourné en hôpital de soins aigus en moyenne deux jours de moins qu'en 2011 avant leur transfert en clinique de réadaptation (différence non significative). La durée de la réadaptation est restée identique pour les patients en réadaptation pulmonaire, et a été réduite d'une journée pour les patients en réadaptation cardiaque. Pour une qualité de vie et un degré d'autonomie inchangés, l'amélioration des performances des patients en réadaptation cardiaque était légèrement inférieure à leur retour à domicile en 2012 par rapport à 2011. Aucune augmentation des renvois ni des complications n'a pu être observée. 
diovaskulären oder pulmonalen Rehabilitation zugewiesen wurden. Für den Einschluss in die Studie mussten sie nach mündlicher und schriftlicher Information für die Erfassung ihrer Daten für Studienzwecke zustimmen. Um den Effekt klimabedingter Faktoren, vor allem bei Patienten mit COPD, zu minimieren, wurden Patienten jeweils in den Monaten Juni bis Dezember eingeschlossen. Ausschlusskriterien waren mangelnde Sprachkenntnisse, um den Fragebogen auszufüllen oder die Patienteninformation zu verstehen.

Die Daten wurden mit dem klinischen Informationssystem und spezifischen Fragebogen erfasst. Registriert wurden die Aufenthaltstage im Akut-

Tabelle 1

Angaben über die Zahlen der eingeschlossenen Patienten pro Gruppe (kardial und pulmonal) und Jahr.

\begin{tabular}{lllll} 
& Pulmonal & \multicolumn{3}{c}{ Kardial } \\
& 2011 & 2012 & 2011 & 2012 \\
\hline Einschluss & 62 & 70 & 65 & 82 \\
\hline Ausschluss & 66 & 77 & 36 & 24 \\
- kein Einverständnis & 11 & 21 & 21 & 16 \\
\hline - fehlendes Sprachverständnis & 11 & 10 & 13 & 8 \\
\hline - kamen nicht aus einer Akutklinik & 44 & 46 & 2 & 0
\end{tabular}

\section{Tabelle 2}

Angaben zu den Charakteristika der Patienten in der pulmonalen Gruppe.

\begin{tabular}{|c|c|c|}
\hline Patientencharakteristika pulmonale Patienten & 2011 n (SD) & $2012 \mathrm{n}(\mathrm{SD})$ \\
\hline Anzahl & 62 & 70 \\
\hline Alter (Jahre) & $71,3(10,7)$ & $69,0(8,8)$ \\
\hline weiblich & 32 & 28 \\
\hline männlich & 30 & 42 \\
\hline $\mathrm{w} / \mathrm{m}$ in $\%$ & $51,7 / 48,3$ & $40,0 / 60,0$ \\
\hline FEV1 & $41 \%(20,1)$ & $44 \%(13,12)$ \\
\hline \multicolumn{3}{|l|}{ GOLD-Stadium } \\
\hline Stadium1 & $3(5 \%)$ & $0(0 \%)$ \\
\hline Stadium2 & $10(16 \%)$ & $18(26 \%)$ \\
\hline Stadium 3 & $18(29 \%)$ & $21(30 \%)$ \\
\hline Stadium4 & $31(50 \%)$ & $31(44 \%)$ \\
\hline \multicolumn{3}{|l|}{ BODE-Index: } \\
\hline Eintritt & $3,97(2,4)$ & $4,0(2,5)$ \\
\hline Austritt & $3,16(2,4)$ & $3,3(2,2)$ \\
\hline \multicolumn{3}{|l|}{ MMRC * } \\
\hline Eintritt & $2,4(1,3)$ & $2,4(1,2)$ \\
\hline Austritt & $1,8(1,3)$ & $1,8(1,1)$ \\
\hline $\mathrm{BMI} \mathrm{kg} / \mathrm{m}^{2}$ & $24,71(6,4)$ & $25,67(7,1)$ \\
\hline Pack years & $52,7(21,5)$ & $48,00(29,8)$ \\
\hline PO2 (mm Hg) bei Eintritt & $61(11,6)$ & $61(9,8)$ \\
\hline \multicolumn{3}{|l|}{$\begin{array}{l}\text { Schweregrad der Komorbidität - } \\
\text { Charlson-Index (0-30 Punkte) }\end{array}$} \\
\hline Punkte bei Eintritt (0-30 Punkte) & $2,54(2,0)$ & $2,30(1,7)$ \\
\hline
\end{tabular}

spital, weiter die Aufenthaltstage in der Rehaklinik, die Dauer der Therapiesitzungen, die Komplikationen, Rückverlegungen ins Akutspital und die Art der weiteren Behandlung nach Austritt aus der Rehabilitationsklinik. Der Schweregrad der Komorbidität wurde mit dem Charlson-Index aus der Diagnoseliste berechnet.

Bei allen Patienten wurden bei Ein- und Austritt folgende Daten erfasst: körperliche Leistungsfähigkeit mit dem 6-Minuten-Gehtest, Mass der Selbständigkeit mit dem Barthel-Index [2], Lebensqualität bei kardialen Patienten mit dem MacNew-Heart-Fragebogen [3] und bei den pulmonalen Patienten mit dem Chronic Respiratory Questionaire (CRQ) [4].

\section{Rehabilitationsprogramm}

Das Programm beinhaltet die vorgegebenen Elemente der nationalen Fachgesellschaften. Der Umfang und die Art des Programms wurden aufgrund des medizinischen Assessments, einer physiotherapeutischen Funktionsdiagnostik und einer Zielformulierung aufgestellt. Die Rehabilitation umfasst ein Gruppenprogramm mit Aktivitäten (Ergometertraining, geführte Wanderungen, Gymnastik, Entspannung) und Schulungselementen (Lebensstilmodifikation, krankheitsspezifische Aufklärung, Ernährungsberatung) sowie Einzeltherapie (Atemtherapie, Mobilisation, Krafttraining, Erlernen und Erstellen eines auf den Patienten angepassten Heimprogrammes).

\section{Analyse der Daten}

Die Patientencharakteristika sind mit Mittelwerten und Standardabweichungen respektive Median und Interquartilabständen, dargestellt. Die Signifikanz der Aufenthaltszeiten und der Unterschied im Gehtest wurde mit dem zweiseitigen T-Test berechnet, die Fähigkeit, Treppen zu steigen, mit dem MannWhitney-Test.

Hauptendpunkt für einen Einfluss von DRG auf die Rehabilitation war eine relevante Verringerung des Gehtests. Angenommen wurde eine Strecke von $35 \mathrm{~m}$ zwischen den kardialen und pulmonalen Differenzen vom Eintritt in die Reha von 2011 zu 2012.

\section{Ethik}

Diese Studie wurde von der kantonalen Ethikkommission des Kantons Aargau bewilligt.

\section{Resultate}

In den Jahren 2011 und 2012 wurden insgesamt 279 Patienten in diese Studie eingeschlossen. Die Auswertung wird für die pulmonale und kardiale Gruppe jeweils getrennt aufgeführt.

\section{Pulmonale Gruppe}

Für die Gruppe mit pulmonaler Rehabilitation wurden 62 Patienten in 2011 und 70 Patienten 2012 aufgenommen. Der Anteil an Männern und Frauen war annähernd gleich. Das Durchschnittsalter der 
Tabelle 3

Angaben über Patientencharakteristika der Gruppe kardialer Patienten, Angaben in Klammern sind Standarddeviationen.

\begin{tabular}{lll}
$\begin{array}{l}\text { Patientcharakteristika } \\
\text { kardiale Patienten }\end{array}$ & 2011 n (SD) $)_{0}$ & 2012 n (SD) \\
\hline Anzahl & 65 & 82 \\
\hline Alter (Jahre) & $66,7(8,8)$ & $66,8(9,0)$ \\
Weiblich & 13 & 15 \\
Männlich & 52 & 67 \\
W/m in \% & $20 / 80$ & $18 / 82$ \\
\hline Anzahl Bypass & $3,2(0,9)$ & $3,3(0,9)$ \\
\hline EF* in \% & $58,0(12,5)$ & $56,5(14,0)$ \\
\hline BMI (kg/m $\left.{ }^{2}\right)$ & $27,4(4,3)$ & $27,9(4,4)$ \\
\hline Pack/Years & $17,6(21,5)$ & $16,5(26,9)$ \\
\hline Charlsons-Index & & $1,4(1,3)$ \\
\hline Punkte bei Eintritt (0-30 Punkte) & $2,4(4,8)$ & \\
*EF: Ejektion Fraction & &
\end{tabular}

Patienten in der Gruppe 2012 verglichen mit 2011 lag um drei Jahre tiefer. Die Werte für die Lungenfunktion, den BMI, das Ausmass der Dyspnoe und den Schweregrad der COPD, gemessen mit dem BODE-Index, waren in den beiden Gruppen vergleichbar. Je ein Teilnehmer in 2011 und 2012 wurde auf eigenen Wunsch früher entlassen, ein Teilnehmer wurde 2012 wegen einer Komplikation (Pleuraerguss) in das Akutspital zurückverlegt.

\section{Kardiale Gruppe}

Für die Gruppe mit kardiovaskulärer Rehabilitation wurden 65 Patienten 2011 und 82 Patienten 2012 aufgenommen. Der Anteil an Männern und Frauen war annähernd gleich. Die Werte für das Alter, die linksventrikuläre Auswurffraktion, den BMI und das Ausmass des Nikotinabusus waren 2011 und 2012 in den beiden Gruppen nahezu gleich. Je ein Teilnehmer von 2011 und 2012 wurde auf eigenen Wunsch früher entlassen, zwei Teilnehmer 2011 ins Akutspi- tal zurückverlegt (wegen Wundinfekten), 2012 ein Teilnehmer (Bypass neu verlegt). Detaillierte Patienteninformationen sind in den Tabellen 1, 2 und 3 dargestellt.

\section{Aufenthaltsdauer im Akutspital und in der Rehabilitationsklinik}

Die Aufenthaltsdauer im Akutspital nahm sowohl bei den kardialen als auch pulmonalen Patienten im Median um zwei Tage ab. Der Unterschied der Mittelwerte ist statistisch nicht signifikant (kardial $\mathrm{p}=0,16$, pulmonal 0,23 ).

\section{Pulmonale Gruppe}

Die mediane Aufenthaltsdauer betrug in den Akutspitälern 16 Tage 2011 (Interquartilabstand, IQR 6 Tage), und 14 Tage in 2012 (IQR 8 Tage). Die Aufenthaltsdauer in der Rehabilitationsklinik veränderte sich nicht und lag 2011 und 2012 bei 21 Tagen, (IQR 7 Tage resp. IQR 4 Tage).

Die Zeiten für Einzeltherapien nahmen im Median bei den pulmonalen Patienten von 2011 auf 2012 deutlich ab (-13\%) von $335 \mathrm{~min}$ auf $280 \mathrm{~min}$ (IQR $140 \mathrm{~min}$ ). Die Gruppentherapiedauern nahmen 2012, verglichen mit 2011, geringgradig zu, um $+5 \%$ auf $1200 \mathrm{~min}$ (IQR $495 \mathrm{~min}$ ).

\section{Kardiale Gruppe}

Die mediane Aufenthaltsdauer betrug in den Akutspitälern 12 Tage 2011 (IQR 6 Tage) und 10 Tage 2012 (IQR 3 Tage). Die Aufenthaltsdauer in der Rehabilitationsklinik war um einen Tag im Median kürzer und lag 2011 bei 21 Tagen (IQR 3 Tage) und 2012 bei 20 Tagen (IQR 2 Tage).

Nahezu gleichbleibend war die Einzeltherapiedauer bei den kardialen Patienten 2011: 230 min (IQR $90 \mathrm{~min}$ ), und 2012: $225 \mathrm{~min}$ (IQR $85 \mathrm{~min}$ ). Die Gruppentherapiedauern nahmen 2012, verglichen mit 2011, geringgradig $\mathrm{zu}, \mathrm{um}+4 \%$ auf $87 \mathrm{~min}$ (IQR $494 \mathrm{~min}$ ) und bei den pulmonalen Patienten um +5\% auf 1200 min (IQR 495 min). Details sind in

Abbildung $1 \mathrm{a}$ und $\mathbf{1 b}$

Darstellung der medianen Aufenthaltsdauer der pulmonalen und kardialen Patienten in den Jahren 2011 und 2012 für die Akutklinik und Rehabilitationsklinik.
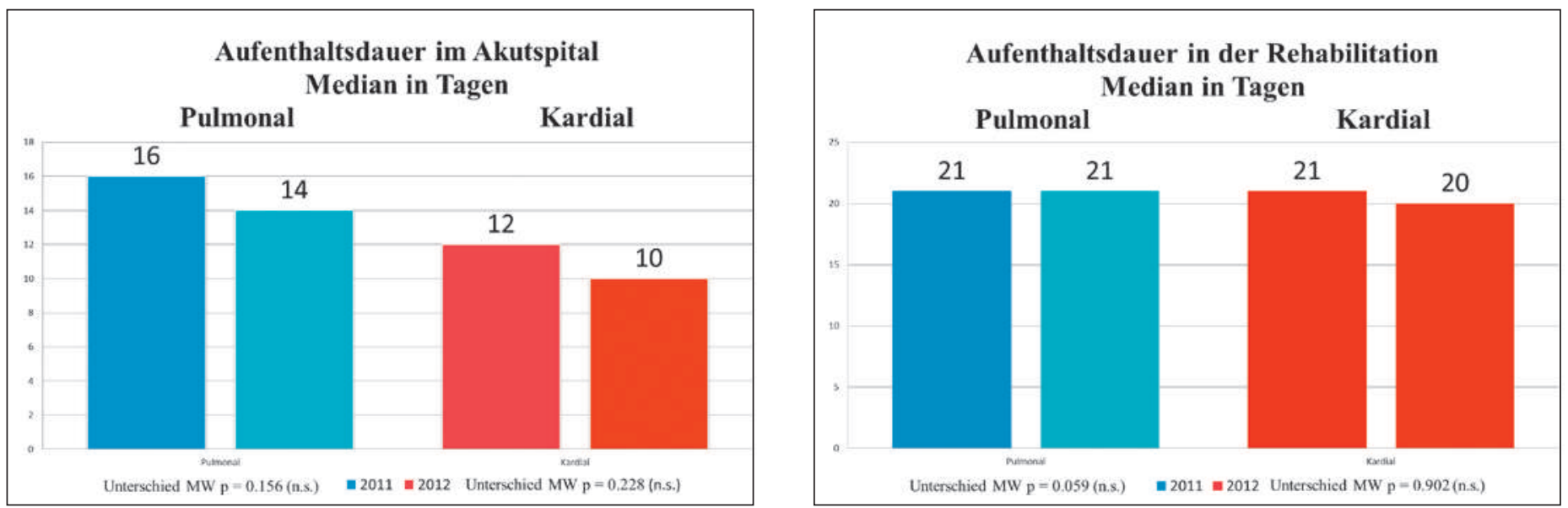


\section{Tabelle 4}

Angaben zur Aufenthaltsdauer in der Akutklinik und Rehabilitationsklinik beider Gruppen. Angaben in Klammern sind Standarddeviationen.

\begin{tabular}{lllll} 
Aufenthaltsdauer akut/Reha (SD) & Pulmonal & \multicolumn{3}{l}{ Kardial } \\
Jahr & 2011 & 2012 & 2011 & 2012 \\
$\begin{array}{l}\text { Aufenthaltsdauer akut; Mittelwert Tage } \\
\text { P-Wert: Unterschied 2011/12 }\end{array}$ & $17,4(5,9)$ & $16,1(6,4)$ & $12,7(4,7)$ & $11,6(4,8)$ \\
Pulmonal: $p=0,156$ kardial: $p=0,228$ & & & & \\
Median & 16 & 14 & 12 & 10 \\
\hline IQR & $14 / 20$ & $12 / 20$ & $8 / 14$ & $9 / 12$ \\
$\begin{array}{l}\text { Aufenthaltsdauer Reha; MW Tage } \\
\text { P-Wert: Unterschied 2011/12 }\end{array}$ & $23,5(6,1)$ & $21,2(6,6)$ & $19,6(3,8)$ & $19,7(3,4)$ \\
Pulmonal: $p=0,059$ kardial: $p=0,902$ & & & & \\
Median & 21 & 21 & 21 & 20 \\
IQR & $20 / 27$ & $19 / 23$ & $18 / 21$ & $19 / 21$
\end{tabular}

den Abbildungen $1 \mathrm{a}$ und $1 \mathrm{~b}$ und auch in Tabelle 4 dargestellt.

\section{Leistungsfähigkeit}

Die körperliche Leistungsfähigkeit wurde mit dem 6-Minuten-Gehtest gemessen. Die angenommene relevante Verringerung des Gehtests um 35 m zwischen den kardialen und pulmonalen Differenzen von Eintritt zu Austritt von 2011 und 2012 wurde nicht erreicht (kardial $-29 \mathrm{~m}$, pulmonal $+18 \mathrm{~m}$; s. Abb. 2 a und 2 b).

\section{Pulmonale Gruppe}

Die Gehstrecke veränderte sich 2011 um $45 \mathrm{~m}$ und 2012 um 63 m (Unterschied 2011/12 n.s.). Allerdings waren die Werte auch bei Eintritt unterschiedlich und lagen 2011 bei $261 \mathrm{~m}$ und in 2012 bei $244 \mathrm{~m}$.

\section{Kardiale Gruppe}

Die Gehstrecke verbesserte sich bei den kardialen Patienten 2011 im Durchschnitt um 126m und 2012 um 97 m (Unterschied 2011/12 n.s.). Die Eintrittsund Austrittswerte waren 2011 und 2012 nahezu gleich. Die Werte bei Ein- und Austritt sind in Tabelle 5 und Abb. 2 a und 2 b dargestellt.

\section{Grad der Selbständigkeit}

Das Mass der Selbständigkeit wurde mit dem Barthel-Index quantifiziert. Der Index gibt auch die Fähigkeit an, Treppen zu steigen.

\section{Pulmonale Gruppe}

Der Durchschnittswert war bei Eintritt 2012 leicht höher als 2011, 5\% der pulmonalen Patienten sind bei Austritt in 2011 keine Treppe gestiegen und 9\% im Jahr 2012.

\section{Kardiale Gruppe}

Der Durchschnittswert war bei Eintritt 2012 tiefer als 2011. Bei diesen Patienten erhöhte sich die Zahl der Patienten signifikant, die vor Eintritt in die Rehabilitation keine Treppen gestiegen sind, von 2011 auf 2012 von 26\% auf 55\% 2012. Detaillierte Angaben sind in Tabelle 5 und Abb. 3 a und 3 b dargestellt.

\section{Lebensqualität}

Sowohl bei den pulmonalen als auch kardialen Patienten verbesserte sich die Lebensqualität während der Rehabilitation.

\section{Pulmonale Gruppe}

Die Lebensqualität war bei den pulmonalen Patienten 2012 bei Ein- und Austritt besser als 2011 (keine signifikanten Veränderungen).

\section{Kardiale Gruppe}

Bei den kardialen Patienten war die Lebensqualität auch 2012 bei Eintritt gleich, und höher bei Austritt als 2011 (keine signifikanten Veränderungen). Siehe hierzu auch Tabelle 5 .

\section{Abbildungen $2 \mathrm{a}$ und $2 \mathrm{~b}$}

Darstellung der Leistungsfähigkeit der pulmonalen und kardialen Patienten 2011 und 2012. Sie zeigt eine verminderte 6-Minuten-Gehtest-Strecke 2012 bei den kardialen Patienten und eine verbesserte bei den pulmonalen Patienten.
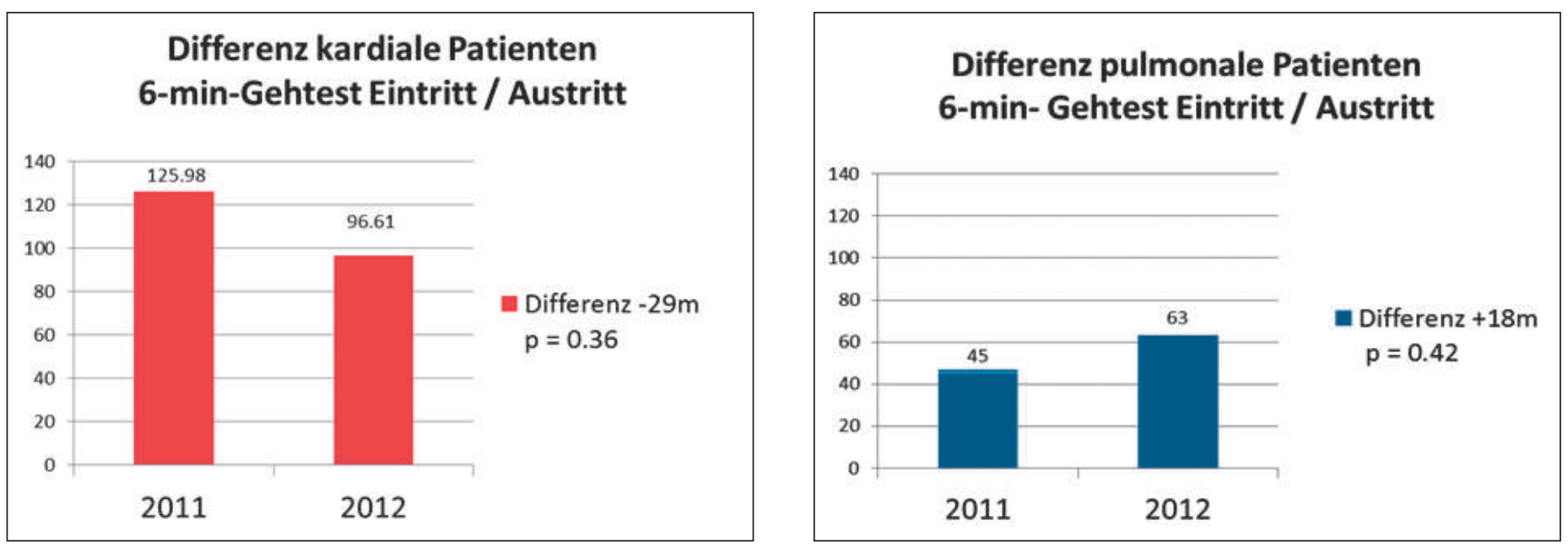
Abbildung $3 a$ und $3 b$

Darstellung des Eintrittszustands der pulmonalen und kardialen Patienten in den Jahren 2011 und 2012. Sie zeigt die prozentuale Verteilung der Patienten, die bei Eintritt keine Treppe gestiegen sind.
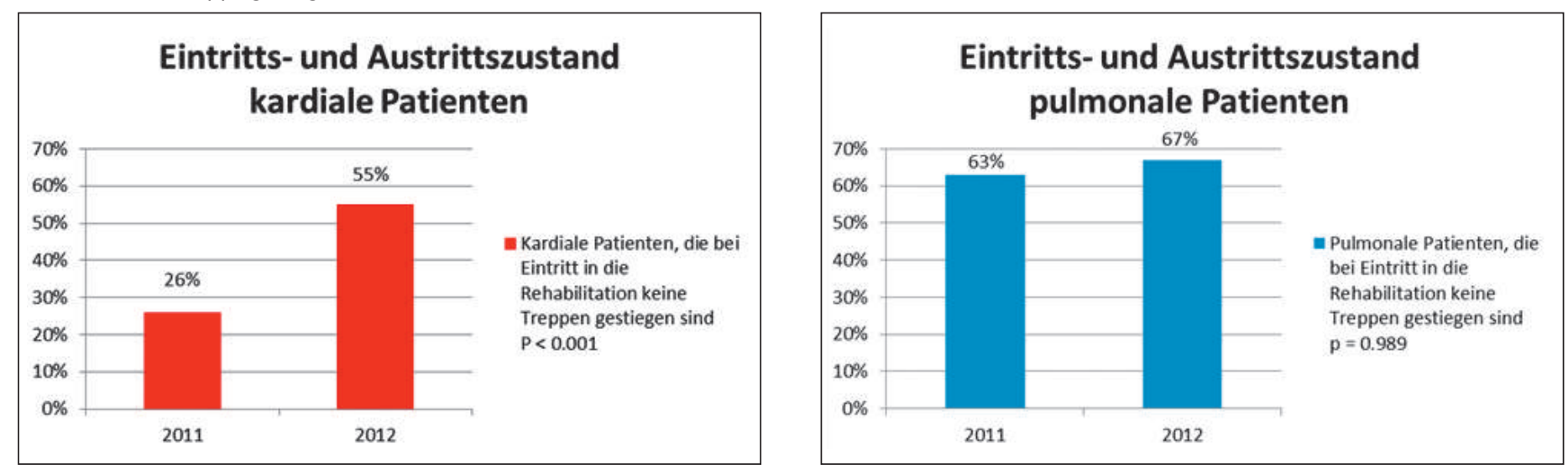

Tabelle 5

Angaben zum Grad der körperlichen Leistungsfähigkeit, dem Grad der Selbständigkeit, und der Lebensqualität bei Ein- und Austritt bei beiden Gruppen. Angaben in Klammern sind Standarddeviationen.

\section{Eintritts- und Austrittszustand}

Jahr

Selbständigkeit - Barthelindex

(0-20 Punkte)

Eintritt

Austritt

keine Treppe

bei Eintritt

bei Austritt

in \% bei Eintritt/ Austritt

p-wert Unterschied, 2011/12

pulmonal $p=0,989$ (n.s.)

kardial $p=0,001$ (s.)

Lebensqualität

\section{CRQ}

$16,2(3,5)$

$18,5(3,1)$

$17,3(2,2)$

$19,1(1,5)$

$18,5(1,6)$

$19,2(2,9)$

$17,6(1,7)$

49/62

$47 / 70$

$17 / 65$

$45 / 82$

$12 / 62$

$11 / 70$

$3 / 65$

$7 / 82$

$63 / 19$

$67 / 16$

$26 / 5$

$55 / 9$

\begin{abstract}
Eintritt
\end{abstract}
$3,2(0,9)$

CRQ

MacNew

MacNew

Austritt

Differenz

$4,3(1,8)$

$3,3(0,8)$

$4,7(1,3)$

$4,7(1,2)$

1,1

1,5

$5,4(1,4)$

$5,7(1,0)$

6-Min.-Gehtest (Meter)

Eintritt

261,0 (128) 244,1 (113) 379,3 (102) 386,4 (116)

Austritt

Differenz
306,3 (122)

45,3
63,3

125,9

\section{Kardiale Gruppe}

Bei den kardialen Patienten von 2011 auf 2012 waren keine Unterschiede feststellbar.

\section{Diskussion}

Diese Studie liefert zwei wichtige Ergebnisse. Erstens, die Aufenthaltsdauer der Patienten im Akutspital verkürzte sich nach Einführung der DRG bei den zur Rehabilitation in die Barmelweid überwiesenen Patienten im Trend um zwei Tage im Median. Das traf sowohl für die kardialen als auch pulmonalen $\mathrm{Pa}-$ tienten zu. Zweitens, der körperliche Leistungszustand der Patienten vor und am Ende der Rehabilitation, gemessen mit dem 6-Minuten-Gehtest, war 2012 nach Einführung der DRG nicht signifikant unterschiedlich zu den Werten im Jahr vor der Einführung. Das gleiche galt für die Lebensqualität und den Bedarf an Nachsorge nach stationärer Rehabilitation.

Bei den kardialen Patienten beobachteten wir in 2012 beim 6-Minuten-Gehtest einen Trend, dass sie sich weniger verbesserten, was zu einer verkürzten Hospitalisationsdauer passen würde. Das bessere Ergebnis bei den pulmonalen Patienten 2012 erklärt sich möglicherweise dadurch, dass diese mit einem tieferen Eingangswert eher einen grösseren Zuwachs an Gehstrecke haben als Patienten mit einem höheren Wert, wie eine Studie von Ferreira zeigte [5].

Auffällig war die Unterschiedlichkeit der Anzahl kardialer Patienten, die während ihres Aufenthaltes im Akutspital nie Treppen gestiegen sind. Die Anzahl Patienten, die während des Akutspitalaufenthaltes noch keine Treppen gestiegen waren, stieg nach Einführung der DRG eindrücklich an. «Treppen steigen» ist für geschwächte Patienten nicht nur eine physische Anstrengung, sondern auch eine psychische Herausforderung, erschwert durch Ängstlichkeit der Patienten. Zudem ist die Fähigkeit, Treppen zu steigen für viele Patienten eine Voraussetzung für den Austritt nach Hause. Mit den in dieser Studie erfassten Daten kann aber die Frage, ob diese 
Veränderung eine direkte Folge der DRG-Einführung ist, nicht beantwortet werden. Es könnte aber ein Hinweis darauf sein, dass in den Akutspitälern die Ressourcen für die Physiotherapie teilweise reduziert wurden.

Die uneinheitlichen Ergebnisse zur Lebensqualität sehen wir in nicht erfassten Confoundern.

Der Charlson-Index deckt im muskuloskelettalen Bereich die Komorbidität nur ungenügend ab. Diese und andere Faktoren könnten bei den Gruppen 2011 stärker ausgeprägt sein, was zu einem geringeren Anstieg der Lebensqualität trotz längerer Verweildauer geführt haben kann.

Wir haben nur eine Studie mit vergleichbarer Fragestellung gefunden. Die REDIA-Studie wurde in Deutschland durchgeführt. Auch dort kam es nach Einführung des DRG-Systems zu einer früheren Verlegung der Patienten aus dem Akutspital in die Rehabilitation.

Die frühere Verlegung macht nach den Worten von Eiffs die Rehabilitation «medizinischer». Er meint damit, dass der Aufwand an Pflege, medizinischen Untersuchungen und Medikamenten zugenommen hat. Zugenommen hat auch die Anzahl der Patienten, die von der Rehabilitationsklinik, meist wegen postoperativen Komplikationen, in ein Akutspital zurücküberwiesen werden mussten. Der funktionelle Austrittszustand der Patienten war gleich wie vor der Einführung [1].

Eine Limitation der Studie ist die beschränkte Anzahl eingeschlossener Patienten. Bis zu 20\%, je nach Gruppe und Jahr, gaben keine Einwilligung, die erfassten Daten in anonymisierter Form für diese Studie zu verwenden. Die teilweise formulierten Befürchtungen, dass Patienten nach Einführung der Finanzierung auf DRG zu schnell vom Akutspital in die Rehabilitation verlegt würden, können mit den Ergebnissen dieser Studie nicht bestätigt werden.

\section{Danksagung}

Dank geht an die Klinikleitung der Klinik Barmelweid für die Unterstützung der Forschungsarbeit und für die finanzielle Unterstützung des Forschungsfonds der Klinik Barmelweid, den Verband physioswiss, den Verband Physiotherapie Rehabilitation und deren Untergruppe kardiale und pulmonale Rehabilitation.

\section{Literatur}

1 von Eiff W, Schüring S, Niehus C. Auswirkung der DRG-Einführung auf die medizinische Rehabilitation. Ergebnisse einer prospektiven medizin-ökonomischen Langzeitstudie 2003-2011. Lit-Verlag; 2011.

2 Mahoney F, Barthel W. Functional evaluation: The barthel index. Md State Med J. 1965;14:61-5.

3 Hofer S, Lim L, Guyatt G, Oldridge N. The macnew heart disease health-related quality of life instrument: A summary. Health Qual Life Outcomes. 2004;2:3.

4 Puhan MA, Behnke M, Frey M, Grueter T, Brändli O, Lichtenschopf A, et al. Self-administration and interviewer-administration of the german chronic respiratory questionnaire: Instrument development and assessment of validity and reliability in two randomised studies. Health Qual Life Outcomes. 2004; 2:1.

5 Ferreira A, Garvey C, Connors GL,Hilling L, Rigler J, Farrell S, et al. Pulmonary rehabilitation in interstitial lung disease: Benefits and predictors of response. Chest. 2009;135(2):442-7.

\section{Interaktiver Artikel}

Wollen Sie diesen Artikel kommentieren? Nutzen Sie dafür die Kommentarfunktion in der OnlineVersion oder sehen Sie nach, was Ihre Kolleginnen und Kollegen bereits geschrieben haben: www.saez.ch/aktuelle-ausgabe/interaktive-beitraege/ 